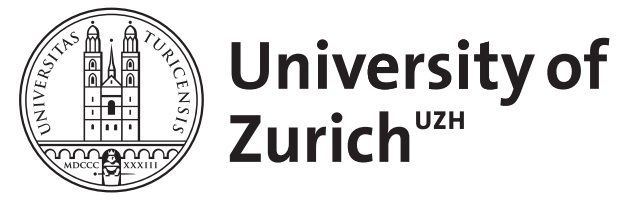

\title{
Adrenal angiomyolipoma in lymphangioleiomyomatosis
}

Sutter, Reto ; Boehler, Annette ; Willmann, Jürgen K

DOI: https://doi.org/10.1007/s00330-006-0206-5

Posted at the Zurich Open Repository and Archive, University of Zurich ZORA URL: https://doi.org/10.5167/uzh-156609

Journal Article

Published Version

Originally published at:

Sutter, Reto; Boehler, Annette; Willmann, Jürgen K (2007). Adrenal angiomyolipoma in lymphangioleiomyomatosis. European Radiology, 17(2):565-566.

DOI: https://doi.org/10.1007/s00330-006-0206-5 


\section{Reto Sutter \\ Annette Boehler \\ Jürgen K. Willmann}

\section{Adrenal angiomyolipoma in lymphangioleiomyomatosis}

Received: 23 January 2006

Accepted: 10 February 2006

Published online: 4 April 2006

(C) Springer-Verlag 2006
R. Sutter · J. K. Willmann ( $\bowtie)$ Institute of Diagnostic Radiology, University Hospital Zurich, Rämistrasse 100 ,

8091 Zurich, Switzerland e-mail: juergen.willmann@usz.ch Tel.: +41-44-2553410

Fax: +41-44-2554443

\section{A. Boehler}

Division of Pulmonary Medicine, University Hospital Zurich,

Zurich, Switzerland
Sir,

A 32-year-old woman with lymphangioleiomyomatosis (LAM) complained of diffuse abdominal pain. Contrast-enhanced multidetector row computed tomography (MDCT) showed a large retroperitoneal, fatcontaining, partially enhancing mass in the right adrenal space and in close contact with the right kidney (Fig. 1). To assess the adrenal localization of the mass, selective intraarterial digital subtraction angiography (DSA) was performed, which demonstrated a vascular supply of the mass through the right inferior suprarenal artery originating from a right accessory renal artery (Fig. 1). After laparoscopic adrenalectomy, an angiomyolipoma of the right adrenal gland was confirmed by histology.

Adrenal angiomyolipoma in LAM is extremely rare. Only one case of a suspected adrenal angiomyolipoma in a patient with LAM has been reported in the literature but was neither documented radiologically nor confirmed by histology [1]. To the best of our knowledge, this is the first report of a histologically proven adrenal angiomyolipoma, documented by MDCT and DSA, in a patient with LAM.

Extrapulmonary manifestations in patients with LAM have been reported in up to $76 \%$ of cases, of which the majority are angiomyolipomas. In most patients, angiomyolipomas are located in the kidneys; rarely, they have been found in the liver [2, 3].
Therefore, with the presence of multiple small angiomyolipomas in both kidneys, it was initially hypothesized that the mass in our patient may be an eccentric cortical manifestation of a large angiomyolipoma in the right kidney with extension into the right adrenal space. However, by demonstrating the arterial supply through the right inferior suprarenal artery, selective DSA proved the adrenal origin of the angiomyolipoma in this case.

Because up to $52 \%$ of patients with angiomyolipomas larger than $4 \mathrm{~cm}$ are symptomatic and have an increased risk of bleeding, surgery or selective arterial embolization has been suggested in large angiomyolipomas. In the last years, laparoscopic adrenalectomy has been recommended because it is less invasive, with lower mortality compared with open surgery.

\section{References}

1. Maziak DE, Kesten S, Rappaport DC, Maurer J (1996) Extrathoracic angiomyolipomas in lymphangioleiomyomatosis. Eur Respir J 9:402-405

2. Avila NA, Kelly JA, Chu SC, Dwyer AJ, Moss J (2000) Lymphangioleiomyomatosis: abdominopelvic CT and US findings. Radiology 216:147-153

3. Kirchner J, Stein A, Viel K, Dietrich CF, Thalhammer A, Schneider M, Jacobi V (1999) Pulmonary lymphangioleiomyomatosis: high-resolution CT findings. Eur Radiol 9:49-54 

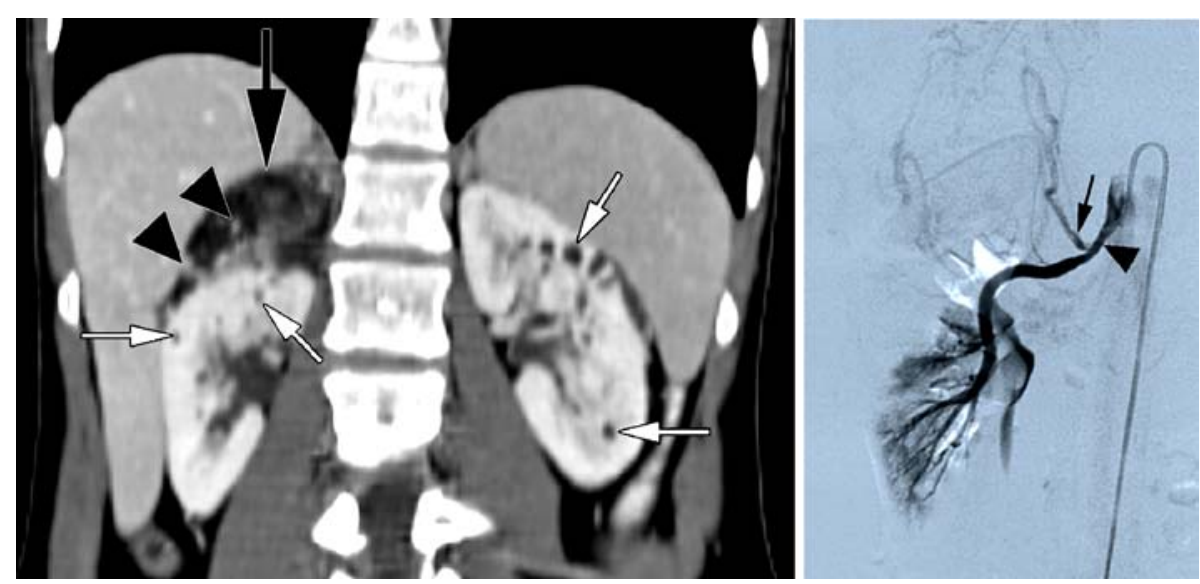

Fig. 1 Coronal multiplanar reconstruction of abdominal contrastenhanced multidetector row computed tomography dataset obtained during the portal-venous phase of the contrast medium shows a large $(6 \mathrm{~cm}$ in maximum transverse diameter) fat-containing mass in the right adrenal space (large arrow) with small enhancing foci (arrowheads). Note multiple small fat-containing angiomyolipomas in both kidneys (small arrows). Selective intraarterial catheter digital subtraction angiography of the right accessory renal artery (arrowhead) demonstrates a well-vascularized adrenal mass supplied by a hypertrophic right inferior suprarenal artery (arrow). Note that the right accessory renal artery also feeds the intermediate renal segment 